\title{
Decay of Gaussian correlations in local thermal reservoirs
}

\author{
Paulina Marian ${ }^{1,2}$, Iulia Ghiu ${ }^{1}$ and Tudor A. Marian ${ }^{1}$ \\ ${ }^{1}$ Centre for Advanced Quantum Physics, Department of Physics, University of \\ Bucharest, R-077125 Bucharest-Măgurele, Romania \\ 2 Department of Physical Chemistry, University of Bucharest, Boulevard Regina \\ Elisabeta 4-12, R-030018 Bucharest, Romania \\ E-mail: paulina.marian@g.unibuc.ro
}

\begin{abstract}
In this paper we examine the decay of quantum correlations for the radiation field in a two-mode squeezed thermal state in contact with local thermal reservoirs. Two measures of the evolving quantum correlations are compared: the entanglement of formation and the quantum discord. We derive analytic expressions of the entanglement-death time in two special cases: when the reservoirs for each mode are identical, as well as when a single reservoir acts on the first mode only. In the latter configuration, we show that all the pure Gaussian states lose their entanglement at the same time determined solely by the field-reservoir coupling. Also investigated is the evolution of the Gaussian quantum discord for the same choices of thermal baths. We notice that the discord can increase in time above its initial value in a special situation, namely, when it is defined by local measurements on the attenuated mode and the input state is mixed. This enhancement of discord is stronger for zero-temperature reservoirs and increases with the input degree of mixing.
\end{abstract}

PACS numbers: 03.67.-a, 03.65.Yz, 03.67.Mn 


\section{Introduction}

The extent to which the non-classical properties of one-mode field states survive in the presence of noise and losses was investigated since the early years of quantum optics [1, 2]. A routine operation like the transmission of light beams through an optical fiber could produce a substantial degradation of their non-classical properties. As an example, it was found that squeezing properties are altered by admixture with thermal noise and disappear completely for values of thermal mean photon occupancy exceeding the threshold $1 / 2$ [3]. From a more recent quantum-information perspective, a lot of work is concentrated on correlations such as entanglement and discord in multipartite systems [4, 5, 6]. While correlations associated with entanglement [4] are defined in connection to global transformations of bipartite quantum states, the concept of quantum discord arises from local (marginal) actions and measurements performed on one subsystem [7, 8]. Its definition contains an optimization over the set of all oneparty measurements, which in the case of mixed states could be a challenging problem. Note that in the pure-state case, entanglement and discord coincide and therefore they measure the total amount of correlations. In the mixed-state case, quantum discord is a measure of quantumness whose relation to entanglement is not a simple one. A survey of recent progress and applications of classical and quantum correlations quantified by quantum discord and other measures can be found in Refs. [5, 6].

When states of a two-party quantum system need to interact with a noisy channel, a drastic modification of their quantum correlations is expected to occur [9, 10]. For instance, it was found that quantum and classical correlations for a system of two qubits evolving in Markovian dephasing channels can display different dynamics [11]. Quite recently, the effect of local noisy channels on quantum correlations in finite-dimensional quantum systems was investigated [12]. It was found that while entanglement does not increase under local channels, other correlations can become larger when the input state is not pure. In the continuous-variable settings, a similar behaviour was recently noticed for the Gaussian discord of two-mode mixed states under single-mode Gaussian dissipative channels [13]. Local Gaussian thermal and phase-sensitive reservoirs modify the entanglement properties of two-mode Gaussian states, as interestingly is pointed out in Refs. [14, 15, 16]. Evolutions of more general Gaussian correlations were also investigated in Refs. [17, 18, 19, 20].

In this work we analyze the decay of quantum correlations of the field in a two-mode Gaussian state due to the interaction of the modes with separate thermal baths. We focus on two measures of quantum correlations, namely, the entanglement of formation (EF) and the quantum discord, and evaluate them for a damped two-mode squeezed thermal state (STS) [21, 22]. Our choice of this important particular class of Gaussian states is motivated by the recent result [23] that for an STS, the exact discord according to its original definition [7, 8] is achieved with an optimal measurement which is Gaussian. Consequently, what was called the Gaussian discord and was derived in Refs. [26, 27] is actually the exact discord. On the other hand, in the interesting paper [28] it 
is shown that for an STS, a Gaussian character of the discord implies that the EF is also Gaussian. Accordingly, the Gaussian EF written explicitly in our paper [29] is equally an exact result. As such, when dealing with a dissipative evolution that preserves the STSs, we have the rare privilege to fully describe the decay of two types of correlations by analytic means.

Our paper is structured as follows. In Section 2 we recapitulate several properties of an STS: the covariance matrix, the Simon separability criterion [24], the entanglement of formation [29] as a measure of inseparability, and the quantum discord as derived in Refs. [26, 27]. In Section 3 the system of interest (field + environment) is specified in order to write and solve the quantum optical master equation. Special attention is then paid to the solution for an input STS. Section 4 deals with a damped STS for modes coupled with two identical local thermal baths. We here derive the time at which the entanglement sudden death occurs and study the evolution of the discord. Section 5 is dedicated to another interesting configuration of the system: only one mode is in contact with a thermal bath. We find that all the pure Gaussian states lose their entanglement at the same time which depends on the reservoir only. In the case of a mixed input state, the discord defined by local measurements on the attenuated mode is increased above its initial value. Our conclusions are drawn in Sec. 6.

\section{Quantum correlations in a two-mode squeezed thermal state}

In this section we review shortly the above-mentioned examples of Gaussian correlations, with emphasis on those of an STS. We consider two-mode Gaussian states and let us denote the photon annihilation operators of the modes by $\hat{a}_{1}$ and $\hat{a}_{2}$. As shown in Refs. [21, 22], an STS is the result of the action of a two-mode squeeze operator,

$$
\hat{S}_{12}(r, \phi):=\exp \left[r\left(\mathrm{e}^{i \phi} \hat{a}_{1}^{\dagger} \hat{a}_{2}^{\dagger}-\mathrm{e}^{-i \phi} \hat{a}_{1} \hat{a}_{2}\right)\right], \quad(r>0, \phi \in(-\pi, \pi]),
$$

on a two-mode thermal state with the mean photon occupancies $\bar{n}_{1}$ and $\bar{n}_{2}$ :

$$
\hat{\rho}_{S T}=\hat{S}_{12}(r, \phi) \hat{\rho}_{T}\left(\bar{n}_{1}, \bar{n}_{2}\right) \hat{S}_{12}^{\dagger}(r, \phi) .
$$

Its covariance matrix (CM) has the following block structure [21, 22]:

$$
\mathcal{V}=\left(\begin{array}{cc}
b_{1} \mathbb{I}_{2} & \mathcal{C} \\
\mathcal{C} & b_{2} \mathbb{I}_{2}
\end{array}\right), \quad\left(b_{1}>\frac{1}{2}, b_{2}>\frac{1}{2}\right) .
$$

In Eq. (2.2), $\mathbb{I}_{2}$ denotes the $2 \times 2$ identity matrix and $\mathcal{C}$ is the $2 \times 2$ symmetric matrix

$$
\mathcal{C}=c\left(\begin{array}{cc}
\cos \phi & \sin \phi \\
\sin \phi & -\cos \phi
\end{array}\right), \quad(c>0) .
$$

Recall that the CM of an STS, Eqs. (2.2) and (2.3), has the standard-form parameters [21, 22]:

$$
b_{1}=\left(\bar{n}_{1}+\frac{1}{2}\right)[\cosh (r)]^{2}+\left(\bar{n}_{2}+\frac{1}{2}\right)[\sinh (r)]^{2}
$$




$$
\begin{aligned}
b_{2} & =\left(\bar{n}_{1}+\frac{1}{2}\right)[\sinh (r)]^{2}+\left(\bar{n}_{2}+\frac{1}{2}\right)[\cosh (r)]^{2}, \\
c & =\left(\bar{n}_{1}+\bar{n}_{2}+1\right) \sinh (r) \cosh (r) .
\end{aligned}
$$

In many applications one can take advantage of a formal definition of an STS, as being an undisplaced and unscaled two-mode Gaussian state described by three standard-form parameters: $b_{1}>\frac{1}{2}, b_{2}>\frac{1}{2}, c>0$. If $b_{1} \geqq b_{2}$, then these parameters must fulfill the uncertainty inequality

$$
\left(b_{1}+\frac{1}{2}\right)\left(b_{2}-\frac{1}{2}\right)-c^{2} \geqq 0 .
$$

If $b_{1}<b_{2}$, then one has to interchange the parameters $b_{1}$ and $b_{2}$ in Eq. (2.5) [22]. The standard form of its CM is given by Eq. (2.2) with the $2 \times 2$ matrix $\mathcal{C}$ written for $\phi=0$, i.e., becoming proportional to the Pauli matrix $\sigma_{3}: \mathcal{C}=c \sigma_{3}$. Within this formal treatment, Eq. (2.1) and its companions, Eqs. (2.2) - (2.4), represent a parametrization of an STS with a clear experimental relevance.

It is known that, according to Williamson's theorem [30], the CM of a two-mode Gaussian state can be diagonalized by a symplectic transformation. We get thus an important ingredient in describing the state, namely, the symplectic eigenvalues of the CM. For an STS they are [22]:

$$
\kappa_{ \pm}=\frac{1}{2}\left[\sqrt{\left(b_{1}+b_{2}\right)^{2}-4 c^{2}} \pm\left(b_{1}-b_{2}\right)\right] .
$$

In the parametrization (2.4), we get $\kappa_{ \pm}=\bar{n}_{1,2}+\frac{1}{2}$.

It is worth mentioning Simon's separability criterion for two-mode Gaussian states [24. It was proven that preservation of the non-negativity of the density matrix under partial transposition is not only a necessary [25], but also a sufficient condition for the separability of two-mode Gaussian states [24]. Accordingly, a two-mode Gaussian state is separable when the condition $\tilde{\kappa}_{-} \geqq \frac{1}{2}$ is met. We have denoted by $\tilde{\kappa}_{ \pm}$the symplectic eigenvalues of the CM corresponding to the partial transpose of the density matrix. For an STS one finds:

$$
\tilde{\kappa}_{ \pm}=\frac{1}{2}\left[b_{1}+b_{2} \pm \sqrt{\left(b_{1}-b_{2}\right)^{2}+4 c^{2}}\right] .
$$

A simplified form of the separability condition for an STS that we shall use in what follows reads [21, 22]:

$$
\left(b_{1}-\frac{1}{2}\right)\left(b_{2}-\frac{1}{2}\right)-c^{2} \geqq 0 .
$$

Before proceeding, let us note that, apart from the vacuum state, the only undisplaced and unscaled pure two-mode Gaussian states are the squeezed vacuum ones, $\left|\psi_{S V}\right\rangle\left\langle\psi_{S V}\right|$, and they belong to the set of STSs. The pure-state case is characterized by the identities $b_{1}=b_{2}=: b$ and $b^{2}-c^{2}=\frac{1}{4}$. Equations (2.6) and (2.7) give now $\kappa_{ \pm}=\frac{1}{2}, \quad \tilde{\kappa}_{ \pm}=b \pm c$.

In the following, we shall recall two measures of quantum correlations for an STS, namely, the EF and the quantum discord. The EF is defined as an optimization over 
all the pure-state decompositions of the given state [32]:

$$
\left.E_{F}(\hat{\rho}):=\inf \left[\sum_{k} p_{k} E\left(\left|\psi_{k}\right\rangle\left\langle\psi_{k}\right|\right)\left|\hat{\rho}=\sum_{k} p_{k}\right| \psi_{k}\right\rangle\left\langle\psi_{k}\right|\right] .
$$

In the expression above, we have denoted by $E\left(\left|\psi_{k}\right\rangle\left\langle\psi_{k}\right|\right)$ the entanglement of the pure bipartite state $\left|\psi_{k}\right\rangle\left\langle\psi_{k}\right|$. We here focus on the case of an STS $\hat{\rho}_{S T}$ and recall that an expression for its EF could be obtained when restricting the optimization in Eq. (2.9) to Gaussian pure-state decompositions only. For further convenience, we introduce the entropic function

$$
h(x):=\left(x+\frac{1}{2}\right) \ln \left(x+\frac{1}{2}\right)-\left(x-\frac{1}{2}\right) \ln \left(x-\frac{1}{2}\right) .
$$

It was proven that the Gaussian EF can be expressed in terms of the function $h(x)$ as:

$$
E_{F}\left(\hat{\rho}_{S T}\right)=h\left(x_{m}\right) \text {. }
$$

In Eq. (2.11), the parameter $x_{m}$ is given in terms of the entries of the CM [29]:

$$
x_{m}=\frac{\left(b_{1}+b_{2}\right)\left(b_{1} b_{2}-c^{2}+\frac{1}{4}\right)-2 c \sqrt{\mathcal{D}}}{\left(b_{1}+b_{2}\right)^{2}-4 c^{2}} .
$$

Here

$$
\mathcal{D}:=\left(b_{1} b_{2}-c^{2}\right)^{2}-\frac{1}{4}\left(b_{1}^{2}+b_{2}^{2}-2 c^{2}\right)+\frac{1}{16} \geqq 0
$$

is the main symplectic invariant. For any squeezed vacuum state, $\mathcal{D}=0$, so that $x_{m}=b$, and thus its EF is equal to the von Neumann entropy of the reduced one-mode thermal state, i. e., $E_{F}\left(\left|\psi_{S V}\right\rangle\left\langle\psi_{S V}\right|\right)=h(b)$.

The difference between two classically equivalent definitions of the mutual information provides another measure of the total amount of quantum correlations in a quantum state, called discord [7, 8]. Let us consider a bipartite state $\hat{\rho}_{A B}$ and write down its quantum mutual information,

$$
I\left(\hat{\rho}_{A B}\right):=S\left(\hat{\rho}_{A}\right)+S\left(\hat{\rho}_{B}\right)-S\left(\hat{\rho}_{A B}\right),
$$

with $S(\hat{\rho})$ being the von Neumann entropy of the state $\hat{\rho}$. Another quantum analogue of the mutual information is more complicated and depends on the influence on the first subsystem $A$ of the measurements made on the second subsystem $B$. Let us denote by $\left\{\hat{\Pi}_{k}^{B}\right\}$ a quantum measurement performed on the system $B$. The final state of the subsystem $A$ after such a measurement on the subsystem $B$ leading to the outcome $j$ is

$$
\hat{\rho}_{A \mid \hat{\Pi}_{j}^{B}}=\frac{1}{p_{j}} \operatorname{Tr}_{B}\left(\hat{\rho}_{A B} \hat{\mathbb{I}}_{A} \otimes \hat{\Pi}_{j}^{B}\right),
$$

In Eq. (2.14),$p_{j}$ is the probability of the outcome $j: p_{j}=\operatorname{Tr}\left(\hat{\rho}_{A B} \hat{\mathbb{I}}_{A} \otimes \hat{\Pi}_{j}^{B}\right)$. The quantum conditional entropy, given the non-selective measurement $\left\{\hat{\Pi}_{j}^{B}\right\}$, is a convex sum of von Neumann entropies of the post-measurement states (2.14) which is taken over all the possible outcomes:

$$
S\left(\hat{\rho}_{A \mid\left\{\hat{\Pi}_{j}^{B}\right\}}\right)=\sum_{j} p_{j} S\left(\hat{\rho}_{A \mid \hat{\Pi}_{j}^{B}}\right) .
$$


The quantum information gained about the subsystem $A$ by taking into account the minimal disturbance produced on it by any of all the possible measurements performed on the subsystem $B$ is the difference [7]

$$
\left.\mathcal{J}\left(\hat{\rho}_{A B}\right)\right|_{\left\{\hat{\Pi}_{j}^{B}\right\}}:=S\left(\hat{\rho}_{A}\right)-\inf _{\left\{\hat{\Pi}_{j}^{B}\right\}} S\left(\hat{\rho}_{A \mid\left\{\hat{\Pi}_{j}^{B}\right\}}\right) .
$$

The quantum $A$-discord is then defined as follows [7]:

$$
D_{1}\left(\hat{\rho}_{A B}\right):=I\left(\hat{\rho}_{A B}\right)-\left.\mathcal{J}\left(\hat{\rho}_{A B}\right)\right|_{\left\{\hat{\Pi}_{j}^{B}\right\}} \geqq 0 .
$$

Similarly, the quantum $B$-discord, which considers the local quantum measurements performed on the first subsystem is

$$
D_{2}\left(\hat{\rho}_{A B}\right):=I\left(\hat{\rho}_{A B}\right)-\left.\mathcal{J}\left(\hat{\rho}_{A B}\right)\right|_{\left\{\hat{\Pi}_{j}^{A}\right\}} \geqq 0 .
$$

Quite recently, the above-defined discord [7, 8] has been calculated for two-mode Gaussian states under the approach of limiting the set of all one-party quantum measurements to the Gaussian ones [26, 27]. We were thus provided with an analytic formula for what is called the Gaussian discord. Moreover, according to Ref. [23], at least for the states analyzed here, namely, the STSs, the Gaussian discord is the exact discord. Thus the quantum discords (2.17) and (2.18) turn out to have very simple expressions in terms of one-mode von Neumann entropies:

$$
\begin{aligned}
& D_{1}^{S T S}=h\left(b_{2}\right)-h\left(\kappa_{+}\right)-h\left(\kappa_{-}\right)+h(y) \\
& D_{2}^{S T S}=h\left(b_{1}\right)-h\left(\kappa_{+}\right)-h\left(\kappa_{-}\right)+h(z) .
\end{aligned}
$$

Here $h$ is the entropic function (2.10) and the symplectic eigenvalues $\kappa_{+}, \kappa_{-}$are given in Eq. (2.6). In addition, we have used the notations:

$$
y:=b_{1}-\frac{c^{2}}{b_{2}+\frac{1}{2}}, \quad z:=b_{2}-\frac{c^{2}}{b_{1}+\frac{1}{2}} .
$$

Note that, for symmetric STSs $\left(b_{1}=b_{2}=: b\right)$, the identity $y=z$ holds and therefore $D_{1}=D_{2}$. Moreover, for pure two-mode Gaussian states, we get $y=z=\frac{1}{2}$ and $D_{1}=D_{2}=h(b)$, i. e., the discord and the entanglement coincide, as expected [5].

\section{Evolution of a two-mode state with two local thermal reservoirs}

We consider an arbitrary two-mode field state having the annihilation operators $\hat{a}_{1}, \hat{a}_{2}$, and the density operator $\hat{\rho}$. Each mode is in contact with a local thermal bath. We denote the mean photon occupancies of the two thermal reservoirs by $\bar{n}_{R j},(j=1,2)$, respectively, and the corresponding damping rates by $\gamma_{j},(j=1,2)$. In the interaction picture, the quantum optical master equation which describes this type of coupling is

$$
\begin{aligned}
& \frac{\partial \hat{\rho}}{\partial t}=\frac{\gamma_{1}}{2}\left(2 \hat{a}_{1} \hat{\rho} \hat{a}_{1}^{\dagger}-\hat{a}_{1}^{\dagger} \hat{a}_{1} \hat{\rho}-\hat{\rho} \hat{a}_{1}^{\dagger} \hat{a}_{1}\right)+\gamma_{1} \bar{n}_{R 1}\left(\hat{a}_{1}^{\dagger} \hat{\rho} \hat{a}_{1}+\hat{a}_{1} \hat{\rho} \hat{a}_{1}^{\dagger}-\hat{a}_{1}^{\dagger} \hat{a}_{1} \hat{\rho}-\hat{\rho} \hat{a}_{1} \hat{a}_{1}^{\dagger}\right) \\
& +\frac{\gamma_{2}}{2}\left(2 \hat{a}_{2} \hat{\rho} \hat{a}_{2}^{\dagger}-\hat{a}_{2}^{\dagger} \hat{a}_{2} \hat{\rho}-\hat{\rho} \hat{a}_{2}^{\dagger} \hat{a}_{2}\right)+\gamma_{2} \bar{n}_{R 2}\left(\hat{a}_{2}^{\dagger} \hat{\rho} \hat{a}_{2}+\hat{a}_{2} \hat{\rho} \hat{a}_{2}^{\dagger}-\hat{a}_{2}^{\dagger} \hat{a}_{2} \hat{\rho}-\hat{\rho} \hat{a}_{2} \hat{a}_{2}^{\dagger}\right) .
\end{aligned}
$$


As in our recent work [31] for the one-mode case, instead of the master equation (3.1), we employ the equivalent differential equation for the two-mode characteristic function $\chi\left(\lambda_{1}, \lambda_{2}, t\right):=\operatorname{Tr}\left\{\left[\hat{D}_{1}\left(\lambda_{1}\right) \otimes \hat{D}_{2}\left(\lambda_{2}\right)\right] \hat{\rho}(t)\right\}$. Here $\hat{D}_{1}\left(\lambda_{1}\right)$ and $\hat{D}_{2}\left(\lambda_{2}\right)$ are the Weyl displacement operators of the modes: $\hat{D}_{j}\left(\lambda_{j}\right):=\exp \left(\lambda_{j} \hat{a}_{j}^{\dagger}-\lambda_{j}^{*} \hat{a}_{j}\right),(j=1,2)$. We finally find the solution:

$$
\begin{aligned}
\chi\left(\lambda_{1}, \lambda_{2}, t\right)= & \chi\left(\lambda_{1} e^{-\frac{1}{2} \gamma_{1} t}, \lambda_{2} e^{-\frac{1}{2} \gamma_{2} t}, 0\right) \exp \left[-\left(\bar{n}_{R 1}+\frac{1}{2}\right)\left(1-e^{-\gamma_{1} t}\right)\left|\lambda_{1}\right|^{2}\right] \\
& \times \exp \left[-\left(\bar{n}_{R 2}+\frac{1}{2}\right)\left(1-e^{-\gamma_{2} t}\right)\left|\lambda_{2}\right|^{2}\right] .
\end{aligned}
$$

Let us inspect the asymptotic behaviour of the solution (3.2) of the master equation (3.1). When we take $t \rightarrow \infty$ in Eq. (3.2), we get the characteristic function of the two-mode thermal state imposed by the two reservoirs:

$$
\lim _{t \rightarrow \infty} \chi\left(\lambda_{1}, \lambda_{2}, t\right)=\exp \left[-\left(\bar{n}_{R 1}+\frac{1}{2}\right)\left|\lambda_{1}\right|^{2}-\left(\bar{n}_{R 2}+\frac{1}{2}\right)\left|\lambda_{2}\right|^{2}\right] .
$$

Note that this two-mode steady state, which is independent of the input state, is a product state without any correlations between the modes.

Given the structure of the time-dependent characteristic function (3.2), any input Gaussian state preserves its Gaussian form at any time during the mode damping. In particular, an initial STS remains an STS at any subsequent time. Its evolving CM has the following standard-form entries:

$$
\begin{aligned}
& b_{1}(t)=b_{1} e^{-\gamma_{1} t}+\left(\bar{n}_{R 1}+\frac{1}{2}\right)\left(1-e^{-\gamma_{1} t}\right), \\
& b_{2}(t)=b_{2} e^{-\gamma_{2} t}+\left(\bar{n}_{R 2}+\frac{1}{2}\right)\left(1-e^{-\gamma_{2} t}\right), \\
& c(t)=c \exp \left[-\frac{1}{2}\left(\gamma_{1}+\gamma_{2}\right) t\right] .
\end{aligned}
$$

In view of Eqs. (3.4), the CM of the damped STS becomes asymptotically diagonal:

$$
\lim _{t \rightarrow \infty} \mathcal{V}(t)=\left(\bar{n}_{R 1}+\frac{1}{2}\right) \mathbb{I}_{2} \oplus\left(\bar{n}_{R 2}+\frac{1}{2}\right) \mathbb{I}_{2}
$$

This means that the two-mode steady state is a product one, whose factors are precisely the single-mode thermal states conditioned by the corresponding reservoirs. Thus we recover the previous general conclusion in the special case of an an initial STS.

To sum up, any measure of correlations in the Gaussian approach available for an STS, such as the entanglement of formation [29] or the quantum discord [26, 27], can readily be applied for a decaying STS on account of Eqs. (3.4).

\section{Evolution of a two-mode squeezed thermal state with two identical local thermal reservoirs}

What are we expecting to occur when a two-mode quantum state is subjected to a dissipative interaction as described by the master equation (3.11)? In general, a 
substantial reduction of the non-classical properties of the state which entails a decrease of its quantum correlations such as entanglement and discord. More specifically, in the important particular case of two-mode Gaussian states, we can notice from the very beginning an important difference between the ways in which these two measures of quantum correlations actually decay. Indeed, on the one hand, according to condition (2.8), the entanglement of the input state is expected to vanish at a finite time. This process has been called the entanglement sudden death in the case of qubits [9, 10]. On the other hand, it is known that the only zero-discord two-mode Gaussian states are the product ones [26, 27]. Taking account of the time-dependent two-mode characteristic function (3.2), as well as of its steady-state form (3.3), we infer that only the latter describes a product state without any correlations between the modes. Therefore, it is reasonable to believe that only asymptotically a damped two-mode Gaussian state could lose all its correlations, both quantum and classical, measured by the Gaussian discord.

For the sake of simplicity and in order to get versatile analytic results, we consider here the particular case when the two local reservoirs are identical: $\gamma_{1}=\gamma_{2}=: \gamma$ and $\bar{n}_{R 1}=\bar{n}_{R 2}=: \bar{n}_{R}$. In this case, the CM of an arbitrary damped two-mode Gaussian state reads:

$$
\mathcal{V}(t)=\mathrm{e}^{-\gamma t} \mathcal{V}(0)+\left(\bar{n}_{R}+\frac{1}{2}\right)\left(1-\mathrm{e}^{-\gamma t}\right) \mathbb{I}_{4}
$$

Here $\mathcal{V}(0)$ is the input $\mathrm{CM}$ and $\mathbb{I}_{4}$ denotes the $4 \times 4$ identity matrix. Equation (4.1) tells us that an input state with no local squeezing does not change its character during damping: for instance, a symmetric state remains symmetric and an STS evolves as a damped STS. We restrict ourselves now to this latter case. When employing the entries of the time-dependent CM (4.1) in the separability condition (2.8), one finds a simple expression of the time required by a damped STS to reach the separability threshold:

$$
t_{s}=\frac{1}{\gamma} \ln \left(1+\frac{\frac{1}{2}-\tilde{\kappa}_{-}}{\bar{n}_{R}}\right), \quad\left(\tilde{\kappa}_{-}<\frac{1}{2}\right) .
$$

Here $\tilde{\kappa}_{-}$is the smallest symplectic eigenvalue of the CM of the partially transposed input density matrix, which is given by Eq. (2.7). We see that in the special case of zero-temperature baths, the entanglement disappears only asymptotically. In all other cases, the quantum-classical transition occurs at finite times, i. e., it happens a sudden death of entanglement [9].

In Figs. 1(a) and 1(b) we plot the evolution of the entanglement of formation, Eq. (2.11), as well as that of the quantum discords $D_{1}$ and $D_{2}$, Eqs. (2.17) and (2.18), for an asymmetric mixed STS. The aspect of the plots follows closely our above remarks on the robustness of discord against noise in comparison with the fragility of entanglement. Note that the discords $D_{1}$ and $D_{2}$ are very close and can be distinguished only for very different values of the thermal mean photon occupancies $\bar{n}_{1}$ and $\bar{n}_{2}$. When the reservoirs are noisy, $\left(\bar{n}_{R}>0\right)$, both the EF and the discords $D_{1}, D_{2}$ are strongly diminished. Contact with zero-temperature baths, as in Fig. 1(b), produces a slower decay of all 

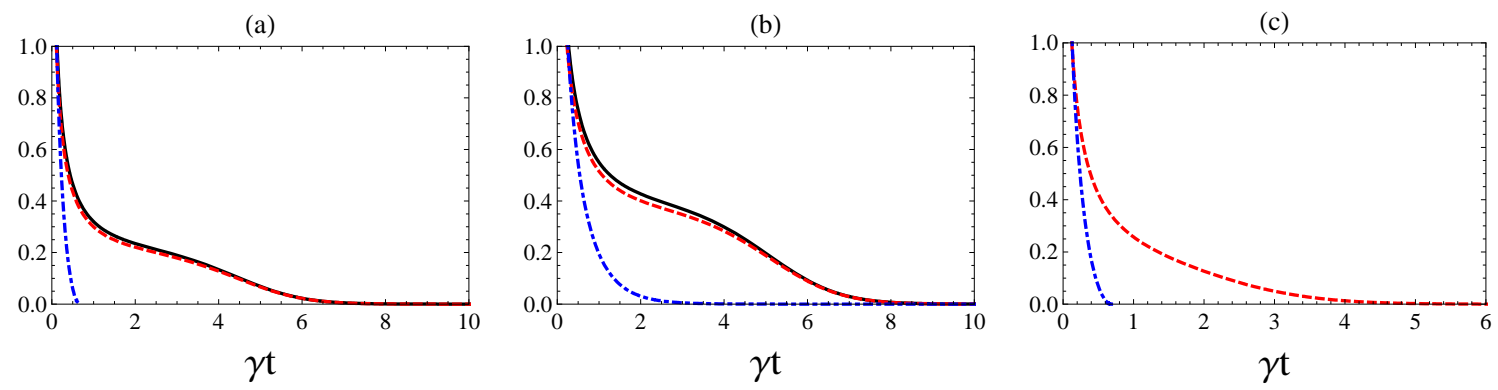

Figure 1. (Color online) Evolution of the EF (dot-dashed blue line), and of the discords $D_{1}$ (black line) and $D_{2}$ (dashed red line) for an input STS in interaction with two local identical thermal reservoirs. We have employed the following parameters. (a) The STS is characterized by the parameters $\bar{n}_{1}=10, \bar{n}_{2}=0.1, r=2$ and the reservoir by $\bar{n}_{R}=0.5$. (b) For the same input state we use $\bar{n}_{R}=0$. (c) We plot the EF and the discord $D_{1}=D_{2}$ (dashed red line) for an input pure state having the squeeze parameter $r=2$. The reservoir is noisy with $\bar{n}_{R}=0.5$.

correlations, which in this case disappear only asymptotically. In Fig. 1(c) we consider an input pure state, namely, a two-mode squeezed vacuum state in contact with a noisy bath. At the time $t=0$ the entanglement and discord coincide, but their time developments look very different. Notice that, in view of Eq. (4.1), a thermalized twomode squeezed vacuum state evolves into a symmetric STS having $D_{1}=D_{2}$.

\section{Evolution of a two-mode squeezed thermal state with a single local thermal reservoir}

For finite-dimensional quantum systems it was recently found that while entanglement does not increase under local channels, other correlations such as discord can become larger when the input state is not pure [12]. In continuous-variable settings, a similar behaviour was noticed for Gaussian discord of mixed two-mode states under one-mode Gaussian dissipative channels [13, 20]. To investigate here such an interaction with analytic means and results, we consider an input STS having only the mode 1 in contact with a thermal reservoir. We specialize the master equation (3.1) to the values $\gamma_{1}=: \gamma, \bar{n}_{R 1}=: \bar{n}_{R}, \gamma_{2}=0, \bar{n}_{R 2}=0$, so that the standard-form entries (3.4) of the damped CM become:

$$
\begin{aligned}
& b_{1}(t)=b_{1} \mathrm{e}^{-\gamma t}+\left(\bar{n}_{R}+\frac{1}{2}\right)\left(1-\mathrm{e}^{-\gamma t}\right), \\
& b_{2}(t)=b_{2}, \\
& c(t)=c \exp \left(-\frac{1}{2} \gamma t\right) .
\end{aligned}
$$

By insertion of the time-dependent parameters (5.1) into the separability condition (2.8) one finds the time at which the EF of a damped STS vanishes:

$$
t_{s}=\frac{1}{\gamma} \ln \left[1-\frac{\left(b_{1}-\frac{1}{2}\right)\left(b_{2}-\frac{1}{2}\right)-c^{2}}{\bar{n}_{R}\left(b_{2}-\frac{1}{2}\right)}\right], \quad\left(b_{1}-\frac{1}{2}\right)\left(b_{2}-\frac{1}{2}\right)-c^{2}<0 .
$$


We specialize Eq. (5.2) to the case of a pure Gaussian input $\left(b_{1}=b_{2}=: b, b^{2}-c^{2}=\frac{1}{4}\right)$. The time of the death of entanglement (5.2) is then independent of the input two-mode squeezed vacuum state, being determined only by the field-reservoir coupling:

$$
t_{c}=\frac{1}{\gamma} \ln \left(1+\frac{1}{\bar{n}_{R}}\right) .
$$

We have also checked that the time of the entanglement death has the same expression (5.3) for an input squeezed vaccuum state with additional local squeezings on both modes. Moreover, in our recent paper [31], we found that, for some classes of onemode states displaying initially certain negativities of their Glauber-Sudarshan $P$ representation, $t_{c}$ is the ultimate time at which the $P$ function becomes positive due to the field interaction with a thermal reservoir. At the time (5.3) it therefore occurs a sudden quantum-classical transition for some types of one-mode states, as well as for any two-mode squeezed vacuum state.

As regards the evolution of the Gaussian discord, we expect it to decay eventually very slowly and to vanish only asymptotically. Indeed, according to Eqs. (5.1), the CM of the damped STS has an asymptotically diagonal form:

$$
\lim _{t \rightarrow \infty} \mathcal{V}(t)=\left(\bar{n}_{R}+\frac{1}{2}\right) \mathbb{I}_{2} \oplus b_{2} \mathbb{I}_{2}
$$

The steady state of the field is therefore the product of two single-mode thermal states: the state of the damped mode 1 , which is imposed by the thermal reservoir owing to their interaction, and that of the freely-evolving mode 2 , which is its reduced state remaining constant in time and thus equal to its input at $t=0$.

The case of a pure-state input deserves additional remarks. According to Eqs. (5.1), although at the moment $t=0$ the three measures of quantum correlations EF, $D_{1}$ and $D_{2}$ coincide, they behave subsequently quite differently because an input twomode squeezed vacuum state evolves into an asymmetric STS. Figure 2(c) displays the evolution of the EF, as well as those of both discords $D_{1}$ and $D_{2}$, which are all monotonic, as predicted in Ref. [12]. However, the discord $D_{2}$ corresponding to local measurements performed on the damped mode 1 survives much longer than both the EF and the discord $D_{1}$.

The case of an initial mixed state can be tackled by using Eqs. (2.11), (2.19), and (5.1) for obtaining the expressions of the EF and the discords $D_{1}$ and $D_{2}$. We plot in Figs. 2(a) and 2(b) their time evolution for the same input state, but with a noisy bath (a) and a zero-temperature reservoir (b). An enhancement of $D_{2}$ is noticed in both panels (a) and (b). The discord $D_{2}$ presents a clear maximum in the latter situation and is much enhanced with respect to its value at the moment $t=0$. This can be interpreted as a creation of quantum correlations similar to those first explored for finite-dimensional systems [12]. Moreover, in the recent Ref. [13] it was found that an enhancement of the discord $D_{2}$ can be noticed even when the input Gaussian state is separable. 
(a)

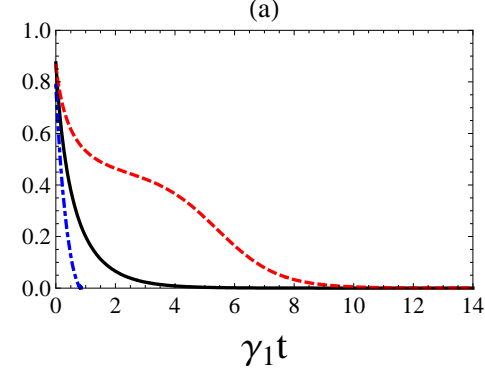

(b)

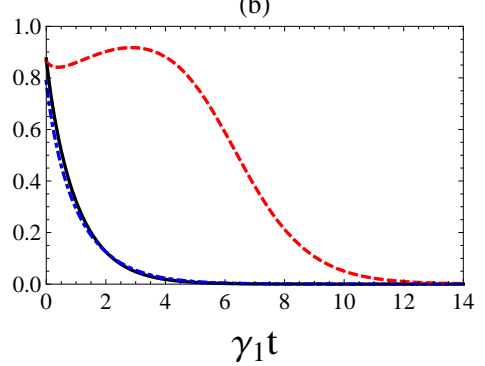

(c)

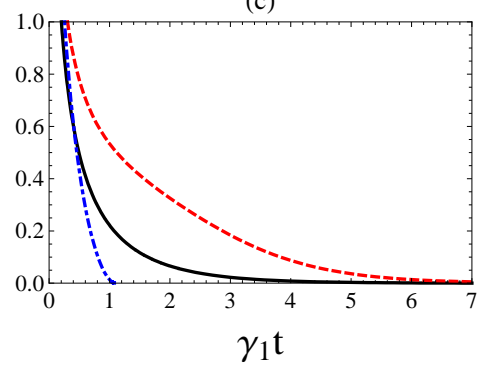

Figure 2. Evolution of the EF (dot-dashed blue line), $D_{1}$ (black line) and $D_{2}$ (dashed red line) in a thermal bath acting on mode 1 . The input state is characterized by the squeeze parameter $r=2$. The input thermal mean photon occupancies are $\bar{n}_{1}=10$, $\bar{n}_{1}=7$ (left and central panels) and the reservoir has (a) $\bar{n}_{R}=0.5$ and (b) $\bar{n}_{R}=0$. The state considered in the panel (c) is pure, while the reservoir has $\bar{n}_{R}=0.5$.

\section{Concluding remarks}

In order to draw some conclusions on the effects produced by local dissipation on quantum correlations of an STS, we compare now the decay of entanglement and discord for the two situations studied above. Figure 3 displays our results for the EF (blue curves) and $D_{2}$ (black plots) in the cases of both one and two local identical reservoirs for a mixed STS (panels (a) and (b)) and for a pure Gaussian state (c). We

(a)

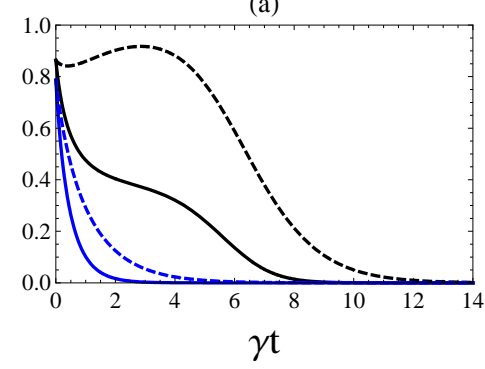

(b)

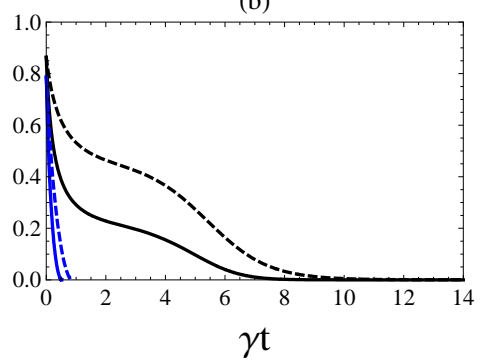

(c)

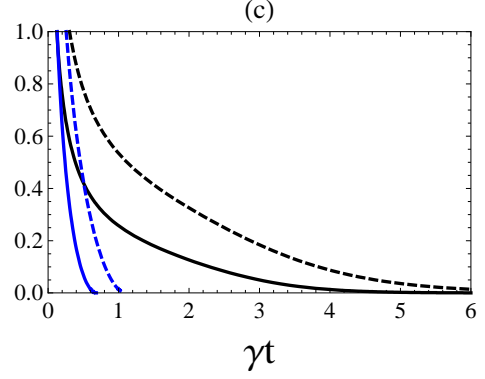

Figure 3. Comparison of decays of the EF (blue curves) and of the discord $D_{2}$ (black curves) for one local bath (dashed lines) and two local identical baths (full lines) for an input state with the squeeze parameter $r=2$. The other parameters are: (a) $\bar{n}_{1}=10$, $\bar{n}_{1}=7, \bar{n}_{R}=0$; (b) $\bar{n}_{1}=10, \bar{n}_{1}=7, \bar{n}_{R}=0.5$; (c) an input pure state and a noisy bath with $\bar{n}_{R}=0.5$.

represent here the case of zero-temperature reservoirs (panels (a) and (c)) to show a better preservation of all correlations in comparison with the noisy bath considered in panel (b). We can see that in all cases both Gaussian discords $D_{1}$ and $D_{2}$ survive longer than the EF. This is expected because only asymptotically the damped Gaussian state becomes a product one. Thus the Gaussian discord, which measures the whole amount of quantum and classical correlations, proves to be quite robust against dissipation in all the above-mentioned situations. However, only for a configuration with one local thermal bath, there is an enhancement of the discord $D_{2}$. Since this can be larger than the discord of the input state, it means that the field-reservoir interaction generates 
quantum and clasical correlations of the discord type. A final conclusion arising from Fig. 3 is quite interesting. In all the analyzed situations (mixed or pure input states, noisy or zero-temperature reservoirs), the configuration with one local thermal bath performs better than that with two local identical baths. This is valid when analyzing both the magnitude of correlations and their preservation in time.

\section{Acknowledgments}

This work was supported by the Romanian National Authority for Scientific Research through Grant PN-II-ID-PCE-2011-3-1012 for the University of Bucharest.

\section{References}

[1] Vourdas A and Weiner R M 1987 Phys. Rev. A 365866

[2] Milburn G J and Walls D F 1988 Phys. Rev. A 381087

[3] Marian P and Marian T A 1996 J. Phys. A: Math. Gen. 296233

[4] Horodecki R, Horodecki P, Horodecki M, and Horodecki K 2009 Rev. Mod. Phys. 81865

[5] Modi K, Brodutch A , Cable H, Paterek T, and Vedral V 2012 Rev. Mod. Phys. 841655

[6] Adesso G, Ragy S, Lee A R 2014 Open Syst. Inf. Dyn. 211440001

[7] Ollivier H and Zurek W H 2001 Phys. Rev. Lett. 88017901

[8] Henderson L and Vedral V 2001 J. Phys. A 346899

[9] Yu T and Eberly J H 2002 Phys. Rev. B 66193306

[10] Maziero J, Céleri L C, Serra R M, and Vedral V 2009 Phys. Rev. A 80044102

[11] Mazzola L, Piilo J and Maniscalco S 2010 Phys. Rev. Lett. 104200401

[12] Streltsov A, Kampermann H, and Bruss D 2011 Phys. Rev. Lett. 107170502

[13] Ciccarello F and Giovannetti V 2012 Phys. Rev. A 85022108

[14] Serafini A, Illuminati F, Paris M G A, and De Siena S 2004 Phys. Rev. A 69022318

[15] Goyal S K and Ghosh S 2010 Phys. Rev. A 82042337

[16] Souza L A M, Drumond R C, Nemes M C, and Fonseca Romero K M 2012 Opt. Commun. 285 4453

[17] Buono D, Nocerino G, Porzio A, Solimeno S 2012 Phys. Rev. A 86042308

[18] Barbosa F A S, de Faria A J, Coelho A S, Cassemiro K N, Villar A S, Nussenzveig P, and Martinelli M 2011 Phys. Rev. A 84052330

[19] Isar A 2012 Phys. Scr. T147 14015

[20] Madsen L S, Berni A, Lassen M, and Andersen U L 2012 Phys. Rev. Lett. 109030402

[21] Marian P, Marian T A, and Scutaru H 2001 J. Phys. A: Math. Gen. 346969

[22] Marian P, Marian T A, and Scutaru H 2003 Phys. Rev. A 68062309

[23] Pirandola S, Spedalieri G, Braunstein S L, Cerf N J, and Lloyd S 2014 Phys. Rev. Lett. 113140405

[24] Simon R 2000 Phys. Rev. Lett. 842726

[25] Peres A 1996 Phys. Rev. Lett. 771413

[26] Giorda P and Paris M G A 2010 Phys. Rev. Lett. 105020503

[27] Adesso G and Datta A 2010 Phys. Rev. Lett. 105030501

[28] Olivares S and Paris M G A 2012 Int. J. Mod. Phys. B 271345024

[29] Marian P and Marian T A 2008 Phys. Rev. Lett. 101220403

[30] Williamson J 1936 Amer. J. Math. 58141

[31] Marian P, Ghiu I, and Marian T A 2013 Phys. Rev. A 88012316

[32] Bennett C H, DiVincenzo D P, Smolin J A, and Wootters W K 1996 Phys. Rev. A 543824 